\title{
Myopericarditis following SARS-CoV-2 mRNA vaccine: the role of cardiac biomarkers and multimodality imaging
}

\author{
Prasanthi Sivakumaran (D) ${ }^{1}$, Juno Sunny ${ }^{1}$, Aliki Tsagkridi ${ }^{2}$, and Mohammed Y. Khanji $\mathbb{D}^{1,2,3 *}$ \\ ${ }^{1}$ Department of Cardiology, Newham University Hospital, Barts Health NHS Trust, Glen Road, Plaistow, London E13 8SL, UK; ${ }^{2}$ Department of Cardiology, Barts Heart \\ Centre, St Bartholomew's Hospital, Barts Health NHS Trust, London EC1A 7BE, UK; and ${ }^{3}$ NIHR Barts Biomedical Research Centre, William Harvey Research Institute, \\ Queen Mary University of London, Charterhouse Square, London EC1M 6BQ, UK \\ *Corresponding author. Tel: +44 207363 8079. E-mail: m.khanji@qmul.ac.uk
}

\begin{abstract}
A 20-year-old male presented with severe chest discomfort associated with palpitations 1-day after his second Pfizer-BioNTech COVID-19 vaccine dose. Other than obesity (body mass index of $32 \mathrm{~kg} / \mathrm{m} 2$ ) he had no other medical conditions and no preceding infective symptoms.
\end{abstract}

Electrocardiogram showed global concave ST-segment elevation suggestive of pericarditis (Panel A). There was a serial rise in troponin (peak 1035ng/L, normal $<14 \mathrm{ng} / \mathrm{L}$ ). Viral screen, including for SARSCoV-2 was negative. There were no arrhythmias on telemetry monitoring. Transthoracic echocardiography revealed low normal left ventricular (LV) systolic function (ejection fraction $50 \%$ ) but no pericardial effusion. Cardiovascular magnetic resonance (CMR) four-chamber view (supplemental video 1) demonstrated elevated lateral LV myocardial T1 (Panel B), T2 (Panel C) and corresponding subepicardial late gadolinium enhancement (LGE, Panel D). Short axis imaging con-

A
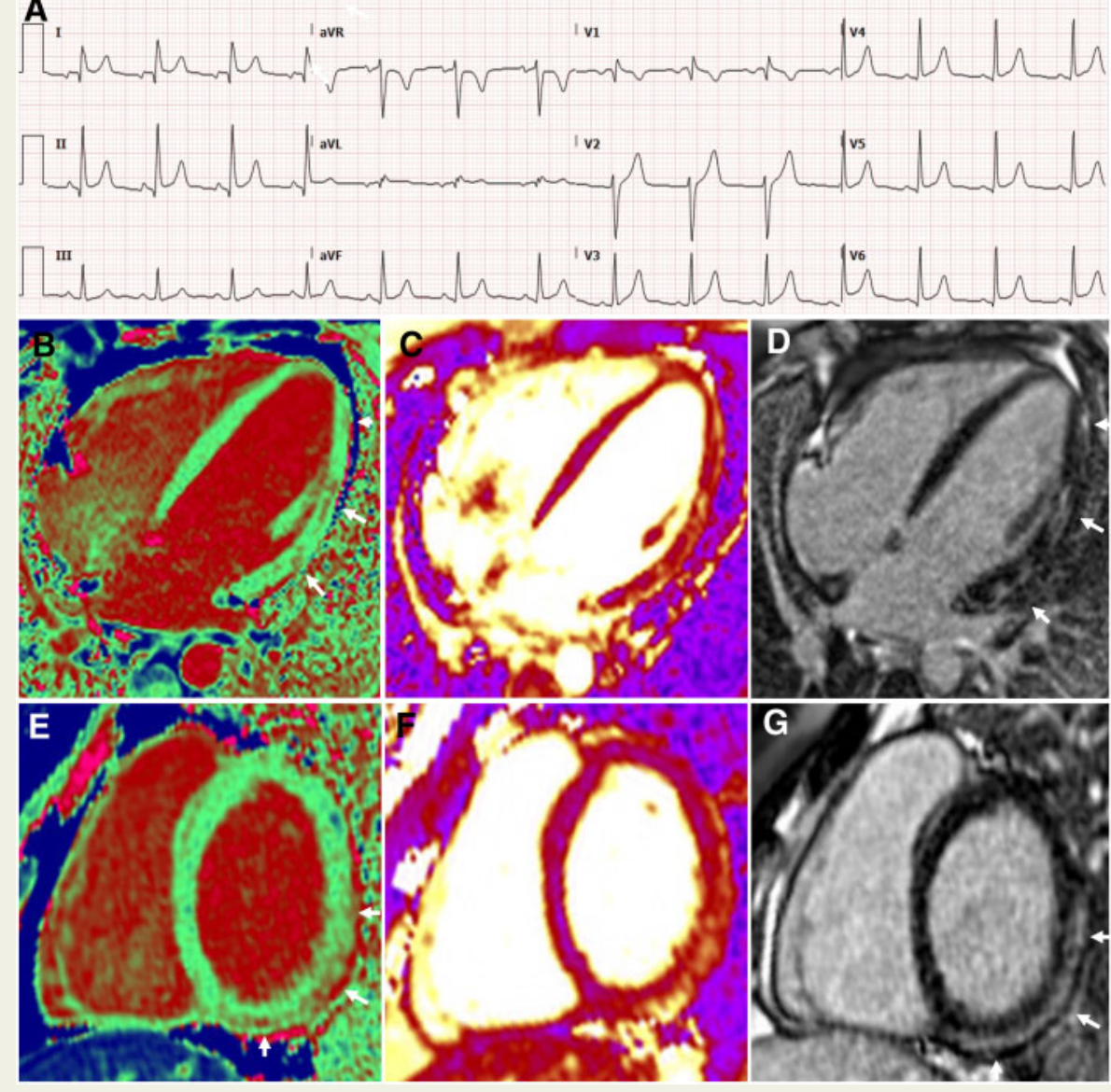

firmed inferior wall involvement

(Panels E, F and G). Once symptoms had improved and troponin levels were decreasing, he was discharge on a 3-month course of colchicine. A repeat CMR in 3-months and further Holter monitoring was arranged.

Cases of myocarditis after SARS-CoV-2 mRNA vaccine in the young population are rare, mild and are typically seen after the second vaccine dose. Presence of long-term consequences are yet to be determined. Differential diagnosis for such presentations such as viral myopericarditis and takotsubo cardiomyopathy should be considered. Serial troponins, echocardiography and multiparametric CMR were pivotal for diagnosis and guiding appropriate management. Overall, the evidence for benefits of reduced hospitalizations and deaths due to COVID-19 following the vaccine are far out-weighed by the potential risks.

Supplementary data are available at European Heart Journal - Cardiovascular Imaging online.

Published on behalf of the European Society of Cardiology. All rights reserved. @ The Author(s) 2021. For permissions, please email: journals.permissions@oup.com. 